\title{
GENERALIZATIONS OF INEQUALITIES OF LITTLEWOOD AND PALEY
}

LOU ZENGJIAN

\author{
Mathematics Depar tment \\ Qufu Normal Unirersity \\ Qufu Shandoag 273165 \\ P. R China
}

(Received July 30, 1991 and in revised form December 13, 1991)

ABSTRACT. For a function $f$, holomorphic in the open unit ball $B_{n}$ in $C^{\infty}$, with $f(0)=0$ we prove

(I) If $0<s<2$ and $s<p<\infty$, Then

$$
|f|_{p}^{p}<C \int_{0}^{1} \int_{B_{0}}|f(\rho \zeta)|^{p-\bullet}|\operatorname{Rf}(\rho \zeta)| \bullet(\log 1 / \rho)^{\infty-1} \rho^{-1} d \sigma(\zeta) d \rho
$$

(II) If $2<8<p<\infty$, Then

$$
\int_{0}^{1} \int_{\partial B_{0}}|f(\rho \zeta)|^{p-\theta}|\operatorname{Rf}(\rho \zeta)|^{\bullet}(\log 1 / \rho)^{\bullet-1} \rho^{-1} d \sigma(\zeta) d \rho<C|f|_{p}^{p}
$$

where $P f$ is the radial derivative of $f$, generalizing the knom caeses $p=B$ (III) and $p=\&, n=1$ ([2]).

KLY WROS AN FHASES Radial derivative slice function.

1991 NS SUBJDCT CLASSIFICATION COAS. 32A10.

\section{Normouction}

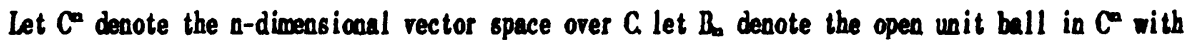
boundary $\partial B^{\circ}$ and let $\sigma$ denote the rotation-invariant positive measure on $\partial B_{0}$ for which $\sigma\left(\partial B_{a}\right)=1$.

Throughout this paper, assume that $f$ is holmorphic in $\mathbb{B}_{\text {n with }} f^{\prime}(0)=0$, and $\operatorname{Rf}(2)=$ $\sum_{\alpha \geq 0}|\alpha| a_{\alpha} z^{\alpha}$ is the radial derirative of $f(z)=\sum_{\alpha>0} \alpha z^{\alpha}$.
For $0<p<\infty$ and $0<8<\infty$ set

$$
\begin{aligned}
& \Delta_{p}(r, f)=\int_{\partial B_{a}}|f(r \xi)| p d \sigma(\zeta) \\
& |f|_{p}=\sup _{0<R_{1}} M_{p}(f, f) \text { and } \\
& G_{p, .}[f]=\int_{0}^{1} \int_{\partial B_{a}}|f(\rho \zeta)|^{p-\bullet}|\operatorname{Rf}(\rho \zeta)| \cdot(\log 1 / \rho) \rho^{-1} \rho^{-1} d \sigma(\zeta) d \rho
\end{aligned}
$$

In [1, Theorem 4 and Theorem 7] J. H Shi generalizes the inequalities of Littlemood and Paley of one cauplex variable ([2]) to the unit ball $\mathrm{B}_{\mathrm{b}}$. That is 
IIEOPEM A (i) Let $0<p<2$. Then

$$
\|f\|_{p}^{p}<C G_{p, p}[f]
$$

(2) Let $2<p<\infty$. Then

$$
G_{p, p}[f]<C\|f\|_{p}^{p}
$$

In this notes, generalize these results, namely, we prove the following

THEPPE (I) Let $0<s<2$ and $8<p<\infty$. Then

$$
\|f\|_{p}^{p}<C G_{p, s}[f]
$$

(II) Let $2<s<p<\infty$. Then

$$
G_{p,} .[f]<C\|f\|_{p}^{p}
$$

Throughout this paper $C$ denotes a positive constant depending only on p and 8 . The magnitude of $C$ mag vary from occurrence to occurrence even in the proof of the same theorem.

\section{PRDO OF THE THEOPOW}

For the proof of the Theorem we need the following

IDMI For $0<p<\infty$. Then

$$
\|f\|_{p}^{p}=p^{2} G_{p, a} \text { [f] }
$$

Procr. For $\zeta \in \partial B_{n}$ the slice functions are defined by $f_{c}\left(\lambda_{0}\right)=f(\lambda \zeta), \lambda \in B_{1}$. Then $\operatorname{Rf}(\lambda \zeta)$ $=\lambda f_{5}(\lambda)$.

By the Hardy_Stein identity for one complex variable ([3]) we have

$$
\begin{aligned}
& \mu \rho\left(r, f_{c}\right)=\left(p^{2} / 2 \pi\right) \int_{0}^{t} \int_{0}^{2 \pi} \mid f_{c}\left(\left.\rho e^{(\theta)}\right|^{p-2} \mid f_{c}\left(\left.\rho e^{(\theta)}\right|^{g} \log (r / \rho) \rho d \rho d \theta\right.\right. \\
& =\left(p^{2} / 2 \pi\right) \int_{0}^{f} \int_{0}^{2 \pi}\left|f\left(\rho \zeta e^{i \theta}\right)\right|^{p-2}\left|\operatorname{Rf}\left(\rho \zeta e^{1 \theta}\right)\right|^{2} \rho^{-1} \log (r / \rho) d \theta d \rho
\end{aligned}
$$

Integrating with respect to $d \sigma(\zeta)$, using the Fubini theorem and the formular

$$
\int_{\partial B^{a}} g(\zeta) d \sigma(\zeta)=(1 / 2 \pi) \int_{\partial B_{e}} d \sigma(\zeta) \int_{0}^{2 \pi} g\left(e^{\prime \theta} \zeta\right) d \theta, \quad g \in L^{1}(\sigma) .
$$

(see [4. P. 16]), we have

$$
\mathscr{L}(r, f)=p^{2} \int_{0}^{r} \int_{\partial B_{p}}|f(\rho \zeta)|^{p-2}|\operatorname{Rf}(\rho \zeta)|^{2} \rho^{-1} \log (r / \rho) d \sigma(\zeta) d \rho
$$

By letting $r \rightarrow 1$ in (6), obtain (5).

We also need the following fact whose easy proof by Holder's inequalityl we mit.

For a fired $p, \log _{\mathrm{g}}$. [ [f] is a convex function of $s\left(0<s<\infty\right.$ ), That is, if $0<s_{1}<8<s_{2}$ $<\infty$ then 


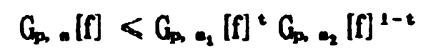

Where $t=\left(\delta_{2}-8\right) /\left(\delta_{2}-s_{1}\right)$.

We now turn to the proof of the Theorm

(I) Case $1.8<p<2$ Set $t=(2-p) /(2-s)$

so that

$$
\begin{array}{rlrl}
\|f\|_{p}^{p} & <C G_{p, p}[f] & (\text { by }(I)) & \\
<C G_{p, ~}[f]^{2} G_{p, ~}[f]^{1-t} & (b y(D)) \\
<C G_{p, ~}[f]^{t} \|\left. f\right|_{p} ^{(a-t)} & (b y(5))
\end{array}
$$

$$
|\mathrm{f}|_{\mathrm{p}}^{p}<\mathrm{CG} \mathrm{G}_{\mathrm{p}, \text { [f] }}
$$

Case 2. $B<2<p$, Set $t=(p-2) /(p-B)$

$$
\begin{aligned}
& \|f\|_{p}^{p}=C_{m, 2}[f] \\
& <C G_{p, a}[f]^{2} G_{p, p}[f]^{1-t} \quad \text { (by (7) ) } \\
& \left.<C G_{p},[f]\right]^{2}\|f\|_{p}^{(a-c)} \quad \text { (by (2) ) }
\end{aligned}
$$

80 that

This gires (3).

$$
|\mathrm{f}|_{p}^{p}<C G_{p h} \text {. [f] }
$$

(II) Set $t=(p-s) /(p-2)$

$$
\begin{aligned}
& G_{p,} .[f]<G_{m, 2}[f]^{t} G_{m p}[f]^{1-t} \quad(\text { by }(7)) \\
& <C|f|_{p}^{p l} G_{p}[f]^{1-2} \quad(\text { by }(E) \text { ) } \\
& <C\|f\|_{p}^{p t} \mid f \|_{p}^{(u-w)} \quad \text { (by (2) ) } \\
& =\mathrm{C} \mid \mathrm{f} \|_{\mathrm{p}}^{\mathrm{p}}
\end{aligned}
$$

This gives (4).

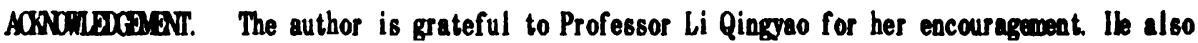
express his thanks to the referee and the Editor Dr. Latenath Debanth for their suggesticas and cooperation.

Porgonas

1. SHI, J. H Hadrmard product of holmorphic functions on unit ball, Chinese Annals of thath $9 \mathrm{~W}$ (1988), 49 _ 69 .

2. LITTEWOCa, J. E and Paley, R E A C. Theorems ou fourier series and pomer series, Proc. Londoo Math. Soc. 12 (1936), 62 _ 89.

3. STEN P. On a theoren of Riesz, J. Loadon Math Soc. 8(1933), 212 _ 217.

4. RUDN W. Function theory in the unit ball of $C^{a}$, Springer_ Ver las New York 1980 


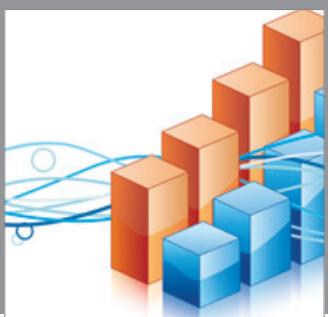

Advances in

Operations Research

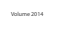

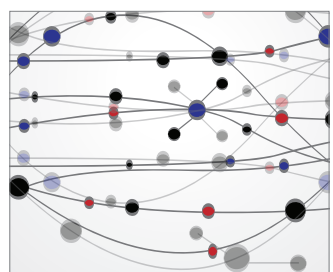

\section{The Scientific} World Journal
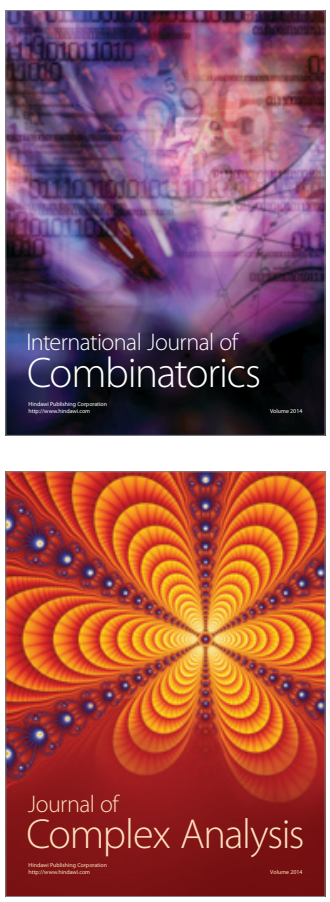

International Journal of

Mathematics and

Mathematical

Sciences
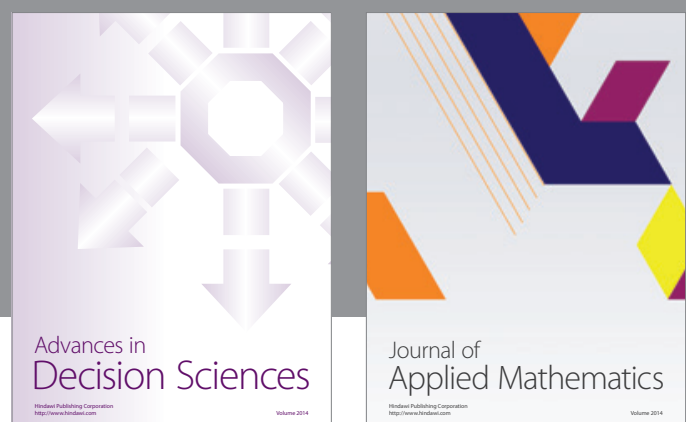

Journal of

Applied Mathematics
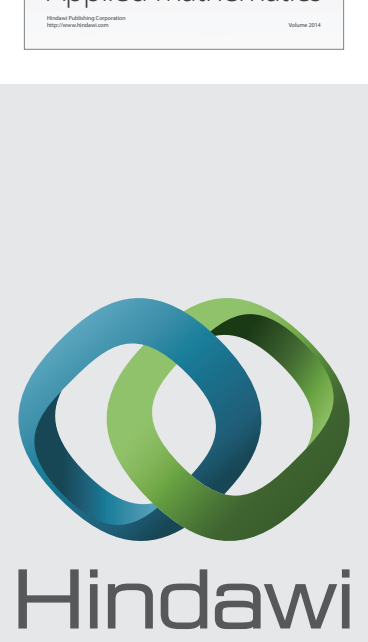

Submit your manuscripts at http://www.hindawi.com
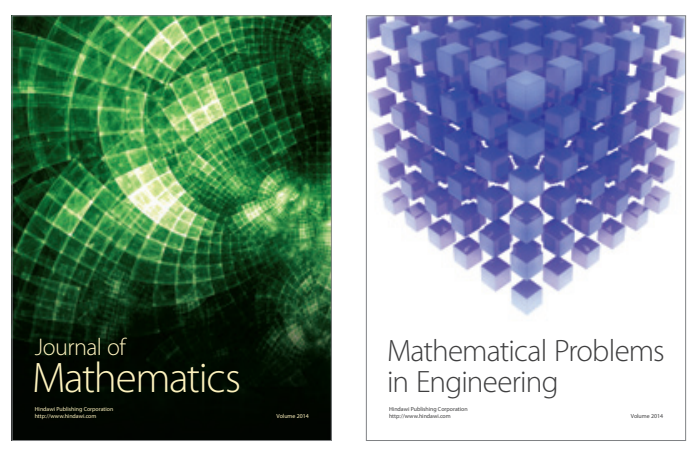

Mathematical Problems in Engineering
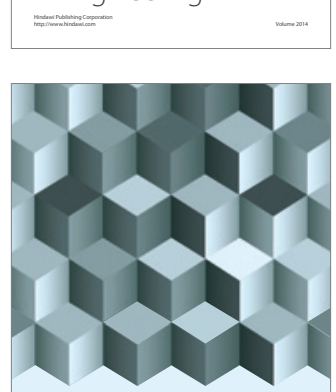

Journal of

Function Spaces
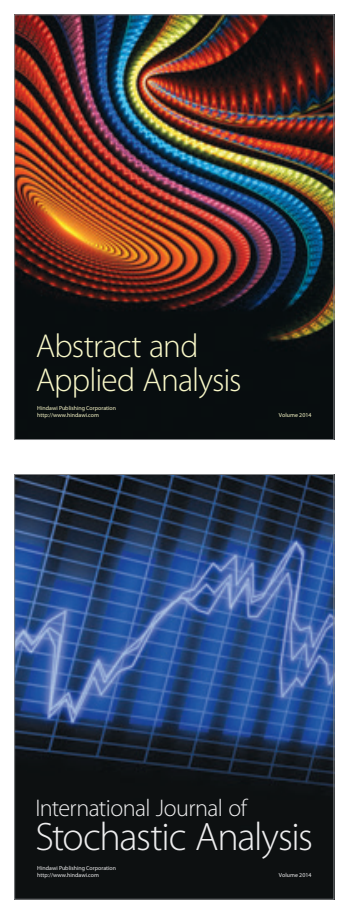

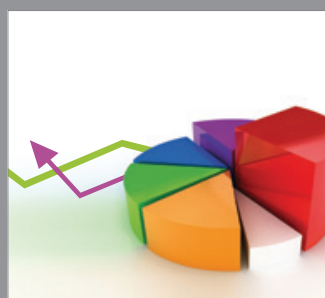

ournal of

Probability and Statistics

Promensencen
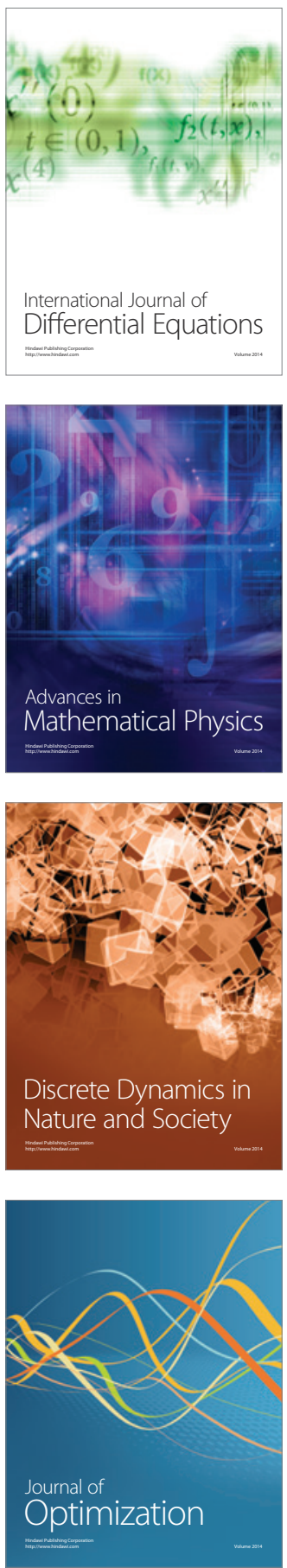